\title{
DELAY DIFFERENTIAL SYSTEMS WITH DISCONTINUOUS INITIAL DATA AND EXISTENCE AND UNIQUENESS THEOREMS FOR SYSTEMS WITH IMPULSE AND DELAY ${ }^{1}$
}

\author{
S.V. KRISHNA \\ Andhra University \\ Department of Mathematics \\ Visakhapatnam, 530003 INDIA \\ A.V. ANOKHIN \\ Chelyabinsk State University \\ Department of Mathematics \\ Chelyabinsk 454138, RUSSIA
}

\begin{abstract}
The main purpose of this paper is to discuss some qualitative aspects of differential equations with delays and impulses. Such systems are encountered in modeling the dynamics of prices and cultured populations. However, any such discussion has to be based on some existence and uniqueness results for delay equations with discontinuous initial data. This is the content of the first part of the paper. For an impulsive system, we observe a phenomenon of existence of infinite number of solutions subject to impulses arbitrarily close to a fixed time. Conditions, when such solutions exist and when they do not, are discussed.
\end{abstract}

Key words: Impulsive delay equations, initial data, continuation.

AMS (MOS) subject classifications: $\quad 34 \mathrm{~A} 37,34 \mathrm{~K} 10$.

\section{INTRODUCTION}

Recently, the theory of impulsive differential equations has gained much attention and popularity, mainly due to the large potential such equations have in providing more realistic models and also due to the mathematical challenges such equations pose. Impulses can change the qualitative (not to mention the quantitative) properties of the solution rather drastically at times (cf. [5]). This can be taken as an advantage, for this suggests the use of impulses as possible

\footnotetext{
${ }^{1}$ Received: August, 1993. Revised: February, 1994.
} 
controls in the dynamics of the state of the system under study.

It is well known that the response of a system of the inputs in real life problems is not instantaneous (delay) and depends on the history of the system. This introduces a delay. Many models in economics, biology, and chemical kinetics fall into this category. An example is a model for the prices of several commodities in a speculative and unscrupulous environment where the customer stocks for speculative reasons and the trader hoards the goods as his utility has reached a threshold value. This model contains both impulses and delays (cf. [4]). Thus the interest in impulsive equations with delays is not just theoretical but practical too.

In the practical situation, the impulses are given at times determined by the solution, and not predetermined. This adds to the difficulties. In this situation, solutions urging for impulses too frequently, even in a small interval of time, is rather ominous. One is also likely to lose the important tool of equating the initial value problem with an integral equation, as will be explained elsewhere [4].

The study of delay differential equations has been largely confined to situations with continuous initial function and when the delay is quite amenable (for example, Volterra delay). But, a more general type of history ought to be considered while dealing with the models and in the presence of impulses, for impulses create discontinuities in the initial function right from the first impulse time.

In this paper we study the existence, uniqueness and pulse control of impulsive differential equations with delay. The existence and uniqueness theorems are in a very general set-up whereas the pulse control is obtained in a much simpler case of Volterra delay. These results can be extended to more general delays with some effort, but we do not attempt this. While proving the existence of a solution of the non-impulsive delay equation, we use an idea of Azbelev [1] which we have used in earlier works [2, 3].

\section{EXISTENCE WITH DISCONTINUOUS INITIAL DATA}

Let $f$ and $h$ satisfy

(C1) $f: \mathbb{R}_{+} \times \mathbb{R}^{n} \rightarrow \mathbb{R}^{n}$ is a Caratheodory function, 
(C2) $h: \mathbb{R}_{+} \rightarrow \mathbb{R}$ is Lebesgue measurable, $h(t) \leq t$ for all $t \in \mathbb{R}_{+}$.

Let $t_{0} \in \mathbb{R}_{+}$. We consider the following initial value problem:

$$
\begin{gathered}
x^{\prime}(t)=f(t, x(h(t))), t>t_{0} \\
x(\sigma)=\varphi(\sigma), \sigma<t_{0},
\end{gathered}
$$

where $\varphi$ is a function, not necessarily a continuous function from $\left(-\infty, t_{0}\right]$ to $\mathbb{R}^{n}$. Our aim is to obtain the existence of a local and global solution of $(2.1,2.2)$, with the initial data $\varphi$ being a discontinuous function and $t_{0}$ any time in $\mathbb{R}_{+}$. We introduce the following operators, which have been first used by Azbelev et al. [1], and later by Anokhin and Krishna [2, 3].

For any function $x:\left[t_{0}, \infty\right) \rightarrow \mathbb{R}^{n}, t_{0} \in \mathbb{R}_{+}$fixed and $\varphi:\left(-\infty, t_{0}\right] \rightarrow \mathbb{R}^{n}$, we define:

and

$$
\left(S\left(h ; t_{0}\right) x\right)(t)=\left\{\begin{array}{cl}
x(h(t)) & \text { if } h(t) \geq t_{0} \\
0 & \text { if } h(t)<t_{0}
\end{array}\right.
$$

$$
\varphi\left(h ; t_{0}\right)(t)=\left\{\begin{array}{cl}
\varphi(h(t)) & \text { if } h(t)<t_{0} \\
0 & \text { otherwise }
\end{array}\right.
$$

We prove the following local existence theorem for the initial value problem $(2.1,2.2)$.

Theorem 2.1: $\quad$ Let $(C 1)$ and $(C 2)$ hold. Assume further

(C3) there exists a Caratheodory function $\omega: \mathbb{R}_{+} \times \mathbb{R}_{+} \rightarrow \mathbb{R}_{+}$such that

(a) $\omega(t, y)$ is nondecreasing in y for almost all $t \in \mathbb{R}_{+}$,

(b) for any $t_{0}, T \in \mathbb{R}_{+}, t_{0}<T$, and for any $y \in \mathbb{R}_{+}, \omega(\cdot, y) \in$ $L_{p}\left[t_{0}, T\right]$, for some $p>1$,

(c) $\|f(t, x)\| \leq \omega(t,\|x\|)$ for $t \in \mathbb{R}_{+}, x \in \mathbb{R}^{n}$.

Then, for any $\left(t_{0}, x_{0}\right) \in \mathbb{R}_{+} \times \mathbb{R}^{n}$, and for any bounded Borel measurable function $\varphi:\left(-\infty, t_{0}\right] \rightarrow \mathbb{R}^{n}$, there exists $\epsilon>0$, such that $(2.1,2.2)$ has an absolutely continuous solution on $\left[t_{0}, t_{0}+\epsilon\right] \subseteq[0, T]$ satisfying the condition

$$
x\left(t_{0}\right)=x_{0} .
$$

Proof: Using (2.3) and (2.4), we can rewrite the initial value problem $(2.1,2.2$ and 2.5$)$ as: 


$$
\left\{\begin{array}{l}
x^{\prime}(t)=f\left(t, \varphi\left(h ; t_{0}\right)(t)+S\left(h ; t_{0}\right) x(t)\right), t \geq t_{0} \\
x\left(t_{0}\right)=x_{0}
\end{array}\right.
$$

which is in turn equivalent to:

$$
x(t)=x_{0}+\int_{t_{0}}^{t} f\left(s, \varphi\left(h, t_{0}\right)(s)+S\left(h, t_{0}\right) x(s)\right) d s .
$$

We introduce the operator $T$ by

$$
(T x)(t)=x_{0}+\int_{t_{0}}^{t} f\left(s, \varphi\left(h, t_{0}\right)(s)+S\left(h, t_{0}\right) x(s)\right) d s
$$

on the space of all absolutely continuous functions $x$ on $\left[t_{0}, T\right], t_{0}<T<\infty$. Then the initial value problem is equivalent to the operator equation

$$
x=T x
$$

on the space of absolutely continuous functions on $\left[t_{0}, T\right]$.

Let $\delta>0$ be fixed, and define $B=\left\{x \in C\left[t_{0}, T\right]: \quad \forall t \in\left[t_{0}, T\right]\right.$, $\left.\left\|x(t)-x_{0}\right\| \leq \delta\right\}$. Treating $T$ as an operator from $C\left[t_{0}, T\right]$ to $C\left[t_{0}, T\right]$, we shall prove that $T B$ is precompact in $C\left[t_{0}, T\right]$. To this end, we observe that for each $x \in C\left[t_{0}, T\right], S\left(h ; t_{0}\right) x$ is a measurable and bounded function on $\left[t_{0}, T\right]$. Hence $S\left(h ; t_{0}\right) x \in L^{\infty}\left[t_{0}, T\right]$. Also, from the definition of $\varphi\left(h ; t_{0}\right)$ and the hypothesis that $\varphi$ is Borel measurable, and $h$ is Lebesgue measurable, it follows that $\varphi\left(h, t_{0}\right)$ is a measurable and bounded function on $\left[t_{0}, T\right]$. Hence $\varphi\left(h ; t_{0}\right) \in L^{\infty}\left[t_{0}, T\right]$ and $\varphi\left(h ; t_{0}\right)+S\left(h ; t_{0}\right) x \in L^{\infty}\left[t_{0}, T\right]$. Also,

$$
\begin{aligned}
& \underset{t \in\left[t_{0}, T\right]}{\operatorname{ess}} \sup \left\|\varphi\left(h ; t_{0}\right)(t)+S\left(h ; t_{0}\right) x(t)\right\| \\
& \underset{t \in\left[t_{0}, T\right]}{\operatorname{esss}} \sup \left\|\varphi\left(h ; t_{0}\right)(t)\right\| \underset{t \in\left[t_{0}, T\right]}{\operatorname{ess}} \sup \left\|S\left(h, t_{0}\right) x(t)\right\| \\
& \leq \kappa_{\varphi}+\left\|x_{0}\right\|+\delta=\kappa\left(\varphi, x_{0}, \delta\right) \text {. }
\end{aligned}
$$

From $(C 1)$ and then $(C 3)(c)$, it follows that $t \mapsto f\left(t, \varphi\left(h ; t_{0}\right)(t)+S\left(h ; t_{0}\right) x(t)\right)$ is measurable and that

$$
\begin{gathered}
\left\|f\left(t, \varphi\left(h ; t_{0}\right)+S\left(h ; t_{0}\right) x(t)\right)\right\| \\
\leq \omega\left(t,\left\|\varphi\left(h ; t_{0}\right)(t)+S\left(h ; t_{0}\right) x(t)\right\|\right) \leq \omega\left(t, \kappa\left(\varphi, x_{0}, \delta\right)\right),
\end{gathered}
$$


where we have used (2.10) and hypothesis $(C 3)(a)$. Further, $(C 3)(b)$ assures that $\omega(\cdot, \kappa) \in L^{p}\left[t_{0}, T\right]$ and hence $t \mapsto f\left(t, \varphi\left(h ; t_{0}\right)(t)+S\left(h ; t_{0}\right) x(t)\right)$ is in $L^{p}\left[t_{0}, T\right]$. Also from (2.8) we have

Consequently, if $x \in B$

$$
\|T x(t)\| \leq\left\|x_{0}\right\|+\int_{t_{0}}^{t} \omega(s, \kappa) d s .
$$

$$
\|T x\|=\sup _{t \in\left[t_{0}, T\right]}\|T x(t)\| \leq\left\|x_{0}\right\|+\int_{t_{0}}^{T} \omega(s, \kappa) d s
$$

which proves that $T B$ is uniformly bounded.

Next, let $t, t^{\prime} \in\left[t_{0}, T\right]$. Then,

$$
\begin{gathered}
\left\|(T x)(t)-(T x)\left(t^{\prime}\right)\right\| \leq\left|\int_{t}^{t^{\prime}}\left\|f\left(s, \varphi\left(h ; t_{0}\right)(s)+S\left(h ; t_{0}\right) x(s)\right)\right\| d s\right| \\
\leq\left|\int_{t}^{t^{\prime}} \omega(s, \kappa) d s\right| \leq\left.\left.\left|t^{\prime}-t\right|^{\frac{p-1}{p}} \cdot\left|\int_{t}^{t^{\prime}}\right| \omega(s, \kappa)\right|^{p} d s\right|^{\frac{1}{p}} .
\end{gathered}
$$

Since by $\omega \in L_{p}\left[t_{0}, T\right]$ by $(C 3)(b)$, the above inequality establishes the equicontinuity of $T B$. Thus $T B$ is precompact in $C\left[t_{0}, T\right]$. Hence corresponding to the $\delta>0$ we have already fixed, there exists $\epsilon>0$ such that for any $x \in B$ (hence for any $T x \in T B),\left(T x\left(t_{0}\right)=x_{0}\right.$ for all $x \in C\left[t_{0}, T\right]$ )

$$
\left\|T x(t)-x_{0}\right\|<\delta
$$

for all $t \in\left[t_{0}, t_{0}+\epsilon\right]$.

We now consider the set $B_{\epsilon}=\left\{x \in C\left[t_{0}, t_{0}+\epsilon\right]:\left\|x(t)-x_{0}\right\| \leq \delta, t \in\right.$ $\left.\left[t_{0}, t_{0}+\epsilon\right]\right\}$. If $x \in B_{\epsilon}$, we can easily find $\bar{x} \in B_{\delta}$, which coincides with $x$ on the segment $\left[t_{0}, t_{0}+\epsilon\right]$, along with its image. That is,

$$
\bar{x}(s)=x(s) \text { for all } s \in\left[t_{0}, t_{0}+\epsilon\right]
$$

and

$$
T \bar{x}(s)=T x(s) \text { for all } s \in\left[t_{0}, t_{0}+\epsilon\right] .
$$

Since $T B$ is precompact in $C\left[t_{0}, T\right]$, from the identification suggested above, it follows that $T B_{\epsilon}$ is precompact in $C\left[t_{0}, t_{0}+\epsilon\right]$ and $T B_{\epsilon} \subseteq B_{\epsilon}$. 
We now apply Schauder's fixed point theorem to the map $T$ on $B_{\epsilon}$ and conclude the existence of a fixed point for $T$ which is a solution on $\left[t_{0}, t_{0}+\epsilon\right]$. Obviously the solution is absolutely continuous on $\left[t_{0}, t_{0}+\epsilon\right]$. This proves the theorem.

In the next theorem, we obtain estimates for the solution of (2.1), (2.2), (2.5) and its growth on the interval of existence which will be used to prove a global existence theorem.

Theorem 2.2: $\quad$ Let $t_{0} \in \mathbb{R}_{+}$and let $(C 1-C 3)$ hold. Suppose that

(C4) there is a $T>t_{0}$ such that for any $y_{0} \in \mathbb{R}_{+}$, the maximal solution of the IVP:

$$
y^{\prime}=\omega(t, y), \quad y\left(t_{0}\right)=y_{0}
$$

exists on $\left[t_{0}, T\right]$. Then for any absolutely continuous solution $x$ of (2.1), (2.2), (2.5) existing on any interval $\left[t_{0}, t_{0}+\epsilon\right] \subseteq\left[t_{0}, T\right]$, the following estimates hold:

$$
\begin{gathered}
\|x(t)\| \leq y\left(t, t_{0},\|\varphi\|+\left\|x_{0}\right\|\right)-\|\varphi\| \\
\|\dot{x}(t)\| \leq \omega\left(t, y\left(t, t_{0},\|\varphi\|+\left\|x_{0}\right\|\right), t \in\left[t_{0}, t_{0}+\epsilon\right],\right.
\end{gathered}
$$

where $\|\varphi\| \sup _{-\infty<t<t_{0}}\|\varphi(t)\|<\infty$ (since $\varphi$ is bounded).

Proof: $\quad$ For any solution $x$ of $(2.1),(2.2),(2.5)$,

$$
\left\|S\left(h ; t_{0}\right) x(t)\right\| \leq \max _{t_{0} \leq s \leq t}\|x(s)\| \leq \| x\left(t_{0}\left\|+\int_{t_{0}}^{t}\right\| \dot{x}(s) \| d s .\right.
$$

We have from (2.1) and the nondecreasing nature of $\omega$, using (2.16)

$$
\begin{gathered}
\|\dot{x}(t)\|=\left\|f\left(t, \varphi\left(h ; t_{0}\right)(t)+S\left(h ; t_{0}\right) x(t)\right)\right\| \\
\leq \omega\left(t,\|\varphi\|+\left\|S\left(h ; t_{0}\right) x(t)\right\|\right), \\
\leq \omega\left(t,\|\varphi\|+\left\|x_{0}\right\|+\int_{t_{0}}^{t}\|\dot{x}(s)\| d s\right) .
\end{gathered}
$$

Let $z(t)=\|\varphi\|+\left\|x_{0}\right\|+\int_{t_{0}}^{t}\|\dot{x}(s)\| d s$. Then $\dot{z}(t)=\|\dot{x}(t)\|$ and

$$
\dot{z}(t) \leq \omega(t, z(t)), \quad z\left(t_{0}\right)=\|\varphi\|+\left\|x_{0}\right\| .
$$


Hence, from (C4) and a well known comparison theorem [6],

$$
\|\dot{x}(t)\|=\dot{z}(t) \leq y\left(t, t_{0},\|\varphi\|+\left\|x_{0}\right\|\right) .
$$

Therefore,

$$
\begin{gathered}
\|\dot{x}(t)\|=\dot{z}(t) \leq \omega(t, z(t)) \\
\leq \omega\left(t, y\left(t, t_{0},\|\varphi\|+\left\|x_{0}\right\|\right)\right) \text { for } t \in\left[t_{0}, t_{0}+\epsilon\right] .
\end{gathered}
$$

Consequently,

$$
\begin{aligned}
\|x(t)\| \leq & \left\|x_{0}\right\|+\int_{t_{0}}^{t}\|\dot{x}(s)\| d s=\left\|x_{0}\right\|+\int_{t_{0}}^{t} \dot{z}(s) d s \\
= & z(t)-z\left(t_{0}\right)+\left\|x_{0}\right\|=z(t)-\|\varphi\| \\
& \leq y\left(t, t_{0},\|\varphi\|+\left\|x_{0}\right\|\right)-\|\varphi\|
\end{aligned}
$$

which proves the theorem.

Theorem 2.3, (Global existence theorem): Assume $(C 1)-(C 4)$. Then for any $t_{0} \in \mathbb{R}_{+}, x_{0} \in \mathbb{R}^{n}$ and $\varphi$ any bounded Borel measurable function on $\left(-\infty, t_{0}\right)$, there exists a solutions $x$ of (2.1), (2.2), (2.5) on $\left[t_{0}, T\right]$.

Proof: Local existence is already established. Suppose that the maximal interval of existence is $\left[t_{0}, t^{*}\right) \subseteq\left[t_{0}, T\right]$. Then, the estimates of Theorem 2.2 show that $\lim _{t \rightarrow t_{-}^{*}} x(t)$ exists and hence the solution can be continued further by Theorem 2.1. This proves global existence.

\section{A UNIQUENESS THEOREM}

The last section establishes existence of an absolutely continuous solution of a system of delay differential equation under very general conditions, particularly with a discontinuous initial data. This is extremely relevant to us as we are bound to encounter such a situation when we deal with impulsive differential equations with delay. However, to make any progress in these difficult situations, we must make some assumptions regarding uniqueness of the non-impulsive solution. In this section we prove such a result, again under a very general hypothesis. 
Theorem 3.1: $\quad$ Suppose $f$ satisfies the condition

$(C 5)$ there exists a function $g:\left[t_{0}, T\right] \times \mathbb{R}_{+} \rightarrow \mathbb{R}_{+}$such that $g$ is Caratheodory, $g(t, 0) \equiv 0, u^{\prime}(t)=g\left(t, u(h(t)), u(\sigma)=0, \sigma \leq t_{0}\right.$ has only zero solution on $\left[t_{0}, t_{0}+\epsilon\right]$ and

$$
\|f(t, x)-f(t, y)\| \leq g(t,\|x-y\|), t \in\left[t_{0}, T\right], x, y \in \mathbb{R}^{n} .
$$

Then, for any bounded Borel measurable $\varphi$ and any $x_{0} \in \mathbb{R}^{n} ;(2.1),(2.2),(2.5)$ has almost one absolutely continuous solution existing on $\left[t_{0}, t_{0}+\epsilon\right] \subseteq\left[t_{0}, T\right]$.

Proof: Follows from Theorem 2.2.

\section{EXISTENCE THEOREMS FOR IMPULSIVE EQUATIONS WITH DELAY}

In this section, we study the existence of solutions for equations with both impulses and delays. Let $\xi: \mathbb{R}_{+} \rightarrow \mathbb{R}^{n}$ and $\tau: \mathbb{R}^{n} \rightarrow \mathbb{R}_{+}, A \subseteq \mathbb{R}_{+} \times \mathbb{R}^{n}, f: \mathbb{R}_{+} \times$ $\mathbb{R}^{n} \rightarrow \mathbb{R}^{n}$ be chosen so as to assure existence and uniqueness of an absolutely continuous solution of the initial value problem (2.1), (2.2), (2.5), for any bounded Borel measurable initial function $\varphi$. We consider the following problem:

$$
\begin{gathered}
x^{\prime}=f(t, x(h(t))), \quad t \geq t_{0} \\
\xi(t) \neq x\left(t_{-}\right), \quad(\text { or } \tau(x(t)) \neq t) \text { or }\left(t, x\left(t_{-}\right)\right) \in \mathcal{A} . \\
\left\{\begin{array}{c}
x(\sigma)=\varphi(\sigma), \quad \sigma<t_{0}, \\
x\left(t_{0}\right)=x_{0}
\end{array}\right. \\
x\left(t_{\xi^{+}}\right)-x\left(t_{\xi^{-}}\right)=\Delta x\left(t_{\xi}\right)=I\left(t_{\xi}, x\left(t_{\xi^{-}}\right)\right)
\end{gathered}
$$

where $I: \mathbb{R}_{+} \times \mathbb{R}^{n} \rightarrow \mathbb{R}^{n}$. This is an impulsive system with delay.

Definition 4.1: A solution of (4.1) to $(4.3)$ on $\left[t_{0}, T\right]$ is a function $x:\left[t_{0}, T\right] \rightarrow \mathbb{R}^{n}$ such that

(1) $x$ is right continuous, and piecewise absolutely continuous on $\left[t_{0}, T\right]$, (i.e., $\left.x \in P A C\left[t_{0}, T\right]\right)$.

(2) Satisfies (4.1) for almost all $t \in\left[t_{0}, T\right]$.

(3) The only points of discontinuity of $x$ are solutions of $x\left(t_{-}\right)=\xi(t)$.

(4) $x$ satisfies (4.3) at these points of discontinuity.

Definition 4.2: We use the following notation: 
$P A C\left[t_{0}, T\right]=\left\{x:\left[t_{0}, T\right] \rightarrow \mathbb{R}^{n}\right.$ such that there exists an absolutely continuous function $y:\left[t_{0}, T\right] \times \mathbb{R}^{n}, \beta_{i} \in \mathbb{R}^{n}, \tau_{i} \in\left[t_{0}, T\right]$ such that

where

$$
\left.x(t)=y(t)+\sum_{i=1}^{\infty} \beta_{i} H_{\tau_{i}}(t)\right\}
$$

$$
H_{\tau_{i}}(t)= \begin{cases}0 & \text { if } t<\tau_{i} \\ 1 & \text { if } t \geq \tau_{i}\end{cases}
$$

is the unit step function.

Remark 1: By demanding the solution to be $P A C\left[t_{0}, T\right]$, and from the definition of the space $P A C\left[t_{0}, T\right]$, it is clear that a solution is necessarily right continuous. This is a deviation, although not a serious one, from the usual definition of solution of an impulsive equation which is taken to be left continuous (cf. [5]).

Remark 2: In the literature so far, impulses were given when the solutions meets a surface of the form $t=\tau(x(t))$ or $\xi(t)=x$. We included here the situation where the impulses may be received when the solution reaches a certain value at a particular time. From an applied point of view this seems to be more reasonable. Also, the earlier results can be recovered by setting $\mathcal{A}=\{(t, x): \xi(t)=x\}$, or $\{(t, x): t=\tau(x)\}$.

Remark 3: An impulse is felt by the solution $x(t)$ only when $\left(t, x\left(t_{-}\right)\right) \in \mathcal{A}$. If $\left(t, x\left(t_{-}\right)\right) \in \mathcal{A}$ and after impulse, $\left.\left(t, x\left(t_{-}\right)\right)+I\left(t, x\left(t_{-}\right)\right)\right) \in \mathcal{A}$, the solution will not receive any impulse, but moves on until it meets $\mathcal{A}$ again. When we discuss the existence of a solution of an I.D.E., we can force the solution of an N.I.D.E. not to meet any impulsive surface $(\tau, \xi$ or $\mathcal{A})$, so that an N.I.D.E. solution is the solution of an I.D.E. also. A number of conditions assuring this can be envisaged from trivial to more sophisticated, but this would be avoiding impulse and so does not become a part of the study of impulsive differential equations. However, we list such conditions to satisfy curiosity.

1) There exists $c>0$ and $\epsilon>0$ such that

$$
\left\|x_{0}-\xi(t)\right\|>c \text { for } t_{0}<t<t_{0}+\epsilon .
$$
2) For $\xi \in C$ and $g(t)=\int_{t_{0}}^{t} f(s, \varphi(s-r)) d s, t \geq 0$, there exists $\epsilon>0$ such
that 


$$
\xi(t)-x_{0} \notin g\left(t_{0}, t_{0}+\epsilon\right) .
$$

3) For $\tau \in C$, there exists $\delta>0$ such that for any $x$ with $\left\|x-x_{0}\right\|<\delta$

$$
\tau(x) \notin g^{-1}\left(x-x_{0}\right) \text {. }
$$

4) For $\xi \in C^{1}$, there exists $\epsilon>0$ such that

$$
\left\{\begin{array}{l}
f(t, \varphi(h(t)))>\xi^{\prime}(t) \text { for almost all } t \in\left[t_{0}, t_{0}+\epsilon\right] \\
\text { or } \begin{array}{l}
f(t, \varphi(h(t)))<\xi^{\prime}(t) \text { for almost all } t \in\left[t_{0}, t_{0}+\epsilon\right]
\end{array}
\end{array}\right.
$$

What is very interesting in this context is, even after assuming the uniqueness of the solution of the N.I.D.E., conditions (4.6) or (4.8) cannot assure the uniqueness of impulsive solutions of I.D.E. as can be seen from the following.

Example:

$$
\begin{gathered}
\dot{x}=1, t \in[0,1], x \neq 2 t, \\
x(0)=0, \\
\left.\Delta x\right|_{x=2 t}=\frac{x}{2}
\end{gathered}
$$

has two solutions, namely $x(t)=t, t \in[0,1]$ and

$$
y(t)=\left\{\begin{array}{cc}
t+e^{-n}, & t \in\left(3^{-n-1}, 3^{-n}\right) \\
0, & t=0 .
\end{array}\right.
$$

The second solution $y$ is extremely interesting. Such solutions will be called singular solutions. Nothing qualitative or quantitative can be said about such solutions, nor do they have good physical interpretations. Thus, isolating the circumstances in which such solutions can occur and do not occur is an important and useful task.

Theorem 4.1: $\quad$ Consider the impulsive system (4.1) to (4.3), with impulses being given to the solution $x$ when $x\left(t_{-}\right)=\xi(t)$. Suppose $I$ and $\xi$ are continuous with $I(0, \xi(0))=0, x_{0}=\xi(0)$. If there exists $a \delta>0$ such that

$$
\left\{x_{0}+\int_{0}^{t} f(s, \varphi(h(s))) d s-\xi(t)\right\} \cdot I(t, \xi(t))>0
$$

for all $t \in(0, \delta)$, then I.D.D.E. (4.1)-(4.3) has a unique solution on $[0, \delta)$, which does not suffer from impulses. 
Theorem 4.2: Consider the I.D.D.E. as in Theorem 4.1, except that (4.9) is replaced by: There exists a $\delta>0$ such that

$$
\left\{x_{0}+\int_{0}^{t} f(s, \varphi(h(s))) d s-\xi(t)\right\} \cdot I(t, \xi(t))<0
$$

and the function

$$
m(t)=\left\{x_{0}+\int_{0}^{t} f(s, \varphi(h(s))) d s-\xi(t)\right\}
$$

is either strictly increasing or decreasing in $[0, \delta)$.

Then the I.D.D.E. has exactly one solution on $[0, \delta)$ which has no impulses and has infinitely many singular (impulsive) solutions. Further, if $\xi$ is assumed to be differentiable, then

$$
\left[f(t, \varphi(h(t)))-\xi^{\prime}(t)\right] \cdot I(t, \xi(t))<0
$$

implies (4.10) and (4.11) and hence the conclusion of the theorem.

Proof of Theorem 4.1: Let $y$ be the unique solution of the (nonimpulsive) D.D.E. Let us denote by $B$ the set $\{t, \xi(t)+I(t, \xi(t)): t \geq 0\}$. From the continuity of $I$ and $\xi$, the hypothesis (4.9) implies that the curves $\{(t, y(t))\}$ and $B$ are on the "same side" of $\{(t, \xi(t))\}$. Hence $y(t)$ does not meet $\xi(t)$ for $t \in(0, \delta]$. This proves the existence of a solution for I.D.D.E. which receives no impulses in $[0, \delta)$.

We shall next prove the uniqueness. Suppose that $x$ is another solution of I.D.D.E. (4.1) to (4.3). Then, it must be an impulsive solution; that is, a solution which receives impulses as it meets $\xi(t)$. It is also clear that 0 must be a limit point of the impulse times of this solution $x$. Let $t_{1}>0$ be a discontinuity of $x$ which means $t_{1}$ is an impulse time of $x$. For definiteness, suppose that $I\left(t_{1}, \xi\left(t_{1}\right)\right)>0$. Then by (4.9) it follows that $x_{0}+\int_{t_{0}}^{t} f(s, \varphi(h(s))) d s-\xi(t)>0$ $t \mapsto I(t, \xi(t))$ is continuous at $t_{1}$. Hence there is $\epsilon$ such that $0<\epsilon<t_{1}$, and there exists a constant $k_{\epsilon}$ such that $I(t, \xi(t))>k_{\epsilon}$ for all $t \in\left(\epsilon, t_{1}\right)$. If in any finite interval, $x$ has an infinite number of discontinuities, then there exists a sequence, $\left\{s_{n}\right\} \subseteq\left\{\epsilon, t_{1}\right)$ of discontinuities of $x$. The jump at each $s_{i}$ is $I\left(s_{i}, x\left(i_{i}-\right)\right)=$ $I\left(s_{i}, \xi\left(s_{i}\right)\right)>k_{\epsilon}$. Since the solution $x \in P A C$, there exists an absolutely continuous function $z$ such that 


$$
x(t)=z(t)+\sum \beta_{i} \chi_{s_{i}}(t), \quad \sum\left|\beta_{i}\right|<\infty,
$$

where $\beta_{i}$ are the jumps of $x$ at the discontinuity times $s_{i}$. Hence,

$$
\beta_{i}=x\left(s_{i}+-x\left(s_{i}-\right)=I\left(s_{i}, \xi_{i}\right)<k_{\epsilon} .\right.
$$

This shows that $\sum \beta_{i}$ diverges. This is a contradiction. Hence on any finite interval, $x$ can have at most a finite number of discontinuities.

Now, let $\delta>0$ be as in the hypothesis, and let the discontinuities of $x$ be $\delta>t_{0}>t_{1}>\ldots>t_{n}>t_{n+1}>\ldots \rightarrow 0$ as $n \rightarrow \infty$. Then we have:

$$
\begin{gathered}
x\left(t_{0}-\right)=x\left(t_{1}-\right)+I\left(t_{1}, \xi\left(t_{1}\right)\right)+\int_{t_{1}}^{t_{0}} f(s, \varphi(h(s))) d s \\
=\xi\left(t_{1}\right)+I\left(t_{1}, \xi\left(t_{1}\right)\right)+\int_{t_{1}}^{t_{0}} f(s, \varphi(h(s))) d s \\
=\ldots \ldots \ldots \\
=\xi\left(t_{k}\right)+\sum_{i=1}^{k} I\left(t_{i}, \xi\left(t_{i}\right)\right)+\int_{t_{k}}^{t_{0}} f(s, \varphi(h(s))) d s
\end{gathered}
$$

for any $k \geq 1$. Since $x\left(t_{0^{-}}\right)=\xi\left(t_{0}\right)$ and $I$ and $\xi$ are continuous and taking the limit of (4.13) as $k \rightarrow \infty$,

$$
\begin{gathered}
\xi\left(t_{0}\right)=x_{0}+\sum_{i=1}^{\infty} I\left(t_{i}, \xi\left(t_{i}\right)\right)+\int_{0}^{t_{0}} f(s, \varphi(h(s))) d s \\
>x_{0}+\int_{0}^{t_{0}} f(s, \varphi(h(s))) d s=x\left(t_{0}-\right)=\xi\left(t_{0}\right) .
\end{gathered}
$$

This is a contradiction. Hence the uniqueness follows.

Remark 4: If the solution receives impulses whenever it meets the curves $\xi_{i}(t), i=1,2, \ldots$, instead of just one curve, the conclusion of Theorem 4.1 can be obtained if (4.9) holds for all $\xi_{i}$ and in addition we have:

If $j$ is the integer such that

$$
I\left(0, \xi_{j}(0)\right)=0, \xi_{j}(0)=x_{0},
$$

then there exists $c>0$ and $\delta>0$ such that 


$$
\left|x_{0}-\xi_{i}(0)\right|>c \text { for all } i \neq j \text {. }
$$

Proof of Theorem 4.2: From (4.10) and (4.11), we may assume, without loss of any generality, that $m(t)>0$ for all $t \in(0, \delta)$. Obviously, then $I(t, \xi(t))<0$ for all $t \in(0, \delta)$. Existence and uniqueness of a non-impulsive solution follow trivially. We shall show that the I.D.D.E. has an infinite number of impulsive solutions existing locally.

Let $[0, T)$ be the interval of existence of the non-impulsive solution $y$. Let $\delta^{\prime}=\min (\delta, T)>0$. Fix $t_{1} \in\left(0, \delta^{\prime}\right)$ and define

$$
x_{1}(t)=\xi\left(t_{1}\right)-\int_{t}^{t_{1}} f(s, \varphi(h(s))) d s, \quad t \in\left[0, t_{1}\right) .
$$

Then $x_{1}$ is differentiable on $\left[0, t_{1}\right)$ and $\dot{x}_{1}(t)=f(t, \varphi(h(t)))$. Thus $x_{1}$ is a solution of the delay differential equation on $\left[0, t_{1}\right)$. Consider the difference $x_{1}(t)-\xi(t)$, for $t \in\left[0, t_{1}\right)$ :

$$
\begin{gathered}
x_{1}(t)-\xi(t)=\xi\left(t_{1}\right)-\int_{t}^{t_{1}} f(s, \varphi(h(s))) d s-\xi(t) \\
=\left[\xi\left(t_{1}\right)-\int_{0}^{t_{1}} f(s, \varphi(h(s))) d s-x_{0}\right]-\left[\xi(t)-\int_{0}^{t} f(s, \varphi(h(s))) d s-x_{0}\right] \\
=m(t)-m\left(t_{1}\right)<0
\end{gathered}
$$

since $m$ is strictly increasing and $t<t_{1}$. Thus $x_{1}(t) \neq \xi(t)$ on $\left(0, t_{1}\right)$.

Let $g(t)=x_{1}(t)-\xi(t)-I(t, \xi(t)), t \in\left[0, t_{1}\right]$. Assume then $g$ is continuous and $g(t)=m(t)-m\left(t_{1}\right)-I(t, \xi(t))$.

We observe that:

$$
\begin{gathered}
g(0)=m(0)-m\left(t_{1}\right)-I(0, \xi(0))=m(0)-m\left(t_{1}\right)<0, \\
g\left(t_{1}\right)=-I\left(t_{1}, \xi\left(t_{1}\right)\right)>0,
\end{gathered}
$$

since we assume $I\left(t_{1}, \xi\left(t_{1}\right)\right)<0$. Hence, there exists $t_{2}, t<t_{2}<t_{1}$ such that $g\left(t_{2}\right)=0 ;$ that is, $x_{1}\left(t_{2}\right)=\xi\left(t_{2}\right)+I\left(t_{2}, \xi\left(t_{2}\right)\right)$. Obviously, $x_{1}$ cannot meet $A$ between $t_{2}$ and $t_{1}$. We now define 


$$
x_{2}(t)=\xi\left(t_{2}\right)-\int_{t}^{t_{2}} f(s, \varphi(h(s))) d s, \quad t \in\left[0, t_{2}\right) .
$$

We proceed as earlier. Repeating this process, we obtain a sequence $\left\{x_{n}\right\}$ of functions and a sequence $\left\{t_{n}\right\} \subset\left[0, t_{1}\right)$ such that:

$$
\begin{gathered}
x_{n}(t)=\xi\left(t_{n}\right)-\int_{t}^{t_{n}} f(s, \varphi(h(s))) d s, \quad t \in\left[0, t_{n}\right) \\
x_{n}\left(t_{n+1}\right)=\xi\left(t_{n+1}\right)+I\left(t_{n+1}, \xi\left(t_{n+1}\right)\right) .
\end{gathered}
$$

Define

$$
x(t)=x_{n}(t), \quad t \in\left[t_{n+1}, t_{n}\right), \quad n=1,2, \ldots
$$

If $t_{N}=0$ for some positive integer $N$, then $x$ is a solution of I.D.D.E. with impulse times $t_{N-1}, \ldots, t_{1}$, and $x(0)=x\left(t_{N}\right)=\xi\left(t_{N}\right)+I\left(t_{N}, \xi\left(t_{N}\right)\right)=x_{0} \quad$ (by hypothesis). If $\left\{t_{n}\right\}$ is infinite, obviously $t_{n}$ decreases to 0 (if $t_{n}$ converges to some non-zero value, $x$ will coincide with the non-impulsive solution which is impossible by the construction of $x$ ).

By the continuity of $\xi$ and $I$, it then follows that

$$
x(0)-\lim _{n} x\left(t_{n}=\lim _{n}\left(\xi\left(t_{n}\right)+I\left(t_{n}, \xi\left(t_{n}\right)\right)\right)=x_{0} .\right.
$$

Hence $x$ is a solution of the I.D.D.E. (4.1) to (.43). We next claim that there are an infinite number of such singular solutions. For, if $t_{1}$ and $t_{1}^{*}$ are in $\left(0, \delta^{\prime}\right)$ with $t_{1}^{*}<t_{1}$, say and if $x_{1}$ and $x_{1}^{*}$ are the two solutions obtained as in the last paragraph, then no impulse point of one can be an impulse point of the other, or else $t_{1}^{*}=t_{1}$. Hence if $t \in\left(0, t_{0}^{*}\right)$, there exist $m$ and $n$ such that

$$
t_{m+1} \leq t \leq t_{m} ; \quad t_{n+1}^{*} \leq t \leq t_{n}^{*}
$$

If $x(t)=x^{*}(t)$, then we should have (after some computations) $m\left(t_{m}\right)=m\left(t_{n}^{*}\right)$ which is not possible since $t_{m} \neq t_{n}^{*}$, and $m$ is strictly increasing. Hence $x$ and $x^{*}$ are different solutions. Lastly, if $\xi$ is differentiable, $m$ is increasing if and only if $m^{\prime}(t)>0$, if and only if $f(t, \varphi(h(t))) \cdot I(t, \xi(t))<0$. This completes the proof of the theorem.

We conclude this section with the following observation. 
A negative result: Let $\xi$ be continuous. Suppose there exists a sequence $\left\{t_{i}\right\}, t_{i} \quad 0$ as $i \rightarrow \infty$ and

$$
\xi_{i}(t)=x_{0}+\int_{0}^{t_{i}} f(s, \varphi(h(s))) d s, \quad i=1,2, \ldots .
$$

If $|I(t, \xi(t))| \geq c>0$ for all $t \in(0, \epsilon)$, for some $\epsilon>0$ and $c>0$, then (4.1) to $(4.3)$ cannot have a solution on any interval $(0, \delta) \subseteq(0, \epsilon)$.

Let $O(t)=\left\{\xi_{i}(t): i=1,2, \ldots,\right\}$ for each $t \in \mathbb{R}_{+}$. For each $\epsilon>0$, let

$$
\begin{aligned}
A_{\epsilon} & =\left\{(t, x) \in[0, \epsilon] \times \mathbb{R}_{+}: x \in O(t)\right\} \\
M_{\epsilon} & =\left\{(t, x) \in[0, \epsilon] \times \mathbb{R}_{+}: x \in \overline{O(t)}\right\} .
\end{aligned}
$$

Then, $A_{\epsilon} \subseteq M_{\epsilon} \subseteq \bar{A}_{\epsilon}$, where - denotes closure.

Proposition: Let there exist $\epsilon>0$ such that

$$
\left(t, x_{0}+\int_{t_{0}}^{t} f(s, \varphi(h(s))) d s\right) \notin M_{\epsilon}, \text { for all } t \in[0, \epsilon) .
$$

Then the I.D.D.E. has a local solution.

\section{CONTINUATION OF THE SOLUTION}

In the last section we discussed the local existence of solution of the I.D.D.E. If the solution of the non-impulsive equation does not meet any impulsive surface, the situation is simple and needs no special attention. If the solution does meet an impulse surface (curve), then Theorem 4.2 gives a condition under which a chaos may develop at the time of impulse. Theorem 4.1 is too strong a negation of Theorem 4.2, since in this theorem, the non-impulsive solution does not meet the impulsive surface for any $t>0$. We would be interested in a result in which the non-impulsive solution does meet an impulsive surface $\xi$ but can continue further. We shall prove such theorems in this section.

Theorem 5.1: Let there exist $p \in L^{1}\left(\mathbb{R}_{+}\right)$and $S: \mathbb{R}_{+} \rightarrow \mathbb{R}_{+}$bounded on bounded subsets of $\mathbb{R}_{+}$such that

$$
\|f(t, x)\| \leq p(t) \cdot S(\|x\|)
$$


for all $t \geq 0$ and $x \in \mathbb{R}^{n}$. Let $h(t) \leq t-r, r>0$ for all $t \geq 0$. Let $\xi$ be continuous with $\xi(0) \neq x_{0}$. Let $I$ be such that for any $T>0$, there exists $c_{1}(T)>0$ with $|I(t, \xi(t))| \geq c_{1}(T)$ for all $t \in[0, T]$. Then, the solution of I.D.D.E. (4.1) to (4.3) can be extended to $\mathbb{R}_{+}$.

Proof: From the hypothesis, local existence is assured. Let $[0, T]$ be the interval of existence. Let $x_{1}$ be the solution. Then for $0 \leq t \leq r$,

$$
x_{1}(t)=x_{0}+\int_{0}^{t} f(s, \varphi(h(s))) d s+\sum_{j} I\left(t_{j}, \xi\left(t_{j}\right)\right)
$$

where $t_{j}$ are such that $x\left(t_{j^{-}}\right)=\xi\left(t_{j}\right)$. Using (5.1)

$$
\left|x_{1}(t)\right| \leq\left|x_{0}\right|+S(\|\varphi\|) \cdot \int_{0}^{t} p(s) d s+\sum_{j}\left|I\left(t_{j}, \xi\left(t_{j}\right)\right)\right| .
$$

We first observe that the summation of the R.H.S. of (5.2) has at most a finite number of terms. To see this, suppose $x$ meets $\xi$ at $t_{i}$, in $[0, r]$. We can, without loss of generality, assume that $t_{i} \rightarrow t^{*} \leq r$ as $i \rightarrow \infty$. Since $\xi$ is continuous, we can find a $\delta>0$ such that for $t, s \in\left(t^{*}-\delta, t^{*}\right),|\xi(t)-\xi(s)|<\frac{c_{1}(r)}{2}$. Hence by hypothesis regarding $I$,

$$
|\xi(t)-I(s, \xi(s))-\xi(s)|>\frac{c_{1}(r)}{2}
$$

for all $t, s \in\left(t^{*}-\delta, t^{*}\right)$. From the convergence of $T_{i}$ to $t^{*}$, there exists a positive integer $N$ s.t. $t_{i} \in\left(t^{*}-\delta, t^{*}\right)$ for all $i \geq N$ and hence,

$$
\left|\xi\left(t_{i+1}\right)-I\left(t_{i}, \xi\left(t_{i}\right)\right)-\xi\left(t_{i}\right)\right|>\frac{c_{1}(r)}{2}, \quad i \geq N .
$$

Let $i \geq N$. Then $x_{1}\left(t_{i+1}-\right)=\xi\left(t_{i+1}\right), x_{1}\left(t_{i}\right)=\xi\left(t_{i}\right)+I\left(t_{i}, \xi\left(t_{i}\right)\right)$ and it follows that:

$$
\left|x_{1}\left(t_{i+1}{ }^{-}\right)-x_{1}\left(t_{i}\right)\right|>\frac{c_{1}(r)}{2} .
$$

On the other hand,

$$
\begin{aligned}
& \left|x_{1}\left(t_{i_{+}}\right)-x_{1}\left(t_{i}\right)\right|=\left|\int_{t_{i}}^{t_{i+1}} f(s, \varphi(h(s))) d s\right| \\
& \quad \leq S(\|\varphi\|) \cdot \sup _{\substack{t \in\left[t_{i}, t_{i+1}\right]}} p(t) \cdot\left(t_{i+1}-t_{i}\right)
\end{aligned}
$$

for all $i \geq N$. Hence, $\left|x_{1}\left(t_{i+1}\right)-x_{1}\left(t_{i}\right)\right| \rightarrow 0$ as $i \rightarrow \infty$, which contradicts (5.3). 
This proves our claim. Thus,

$$
\left|x_{1}(t)\right| \leq\left|x_{0}\right|+S(\|\varphi\|) \int_{0}^{t} p(s)+\sum_{0 \geq t_{j} \leq r}\left|I\left(t_{j}, \xi\left(t_{j}\right)\right)\right|=B_{1} .
$$

We note that $B_{1}$ is a constant depending only on $x_{0}, \varphi, h, I$ and $\xi$. For $t \in[r, 2 r]$, we have

$$
\begin{aligned}
x_{2}(t) & =x_{0}+\int_{t_{0}}^{r} f(s, \varphi(h(s))) d s+\sum_{0 \leq t_{j}<r} I\left(t_{j}, \xi\left(t_{j}\right)\right) \\
& +\int_{r}^{t} f\left(s, x_{1}(h(s))\right) d s+\sum_{r \geq t_{j} \leq 2 r} I\left(t_{j}, \xi\left(t_{j}\right)\right) .
\end{aligned}
$$

We have, using (5.4)

$$
\left|x_{2}(t)\right| \leq B_{1}+S\left(B_{1}\right) \int_{r}^{2 r} p(s) d s+\sum_{r \geq t_{j} \leq 2 r}\left|I\left(t_{j}, \xi\left(t_{j}\right)\right)\right|=B_{2} .
$$

The constant $B_{2}$ depends on $B_{1}, r, I, h$ and $\xi$. Since, $B_{1}$ itself depends on $\varphi$ and $x_{0}, B_{2}$ depends on $\varphi, x_{0}, r, h, \xi$ and $I$. By induction, we conclude, that for any $t \in[k r,(k+1) r]$ and for any positive integer $k$, there exists a constant $B_{k}\left(\varphi, x_{0}, h, \xi, I\right)$ such that

$$
\left|x_{K}(t)\right| \leq B_{k}
$$

Hence for any $T>0$,

$$
\left|x_{1}(t)\right| \leq B\left(T, \varphi, x_{0}, h, \xi, I\right) \text { for all } t \in[0, T] .
$$

Further, if $0<t<t^{\prime}<T$, then

$$
\left|x_{1}(t)-x_{1}\left(t^{\prime}\right)\right|=S(B(T)) \cdot \int_{t}^{t^{\prime}} p(s) d s+\sum_{t<t_{i}<t^{\prime}}\left|I\left(t_{i}, \xi\left(t_{i}\right)\right)\right| .
$$

Hence as $t-t^{\prime} \rightarrow 0, \quad\left|x_{1}(t)-x_{1}\left(t^{\prime}\right)\right| \rightarrow 0 . \quad$ As we can find a left neighborhood of $T$ in which $x$ has no discontinuity, from (5.6) we conclude that $x_{1}\left(T_{-}\right)$exists. If $x_{1}\left(T_{-}\right) \neq \xi(T)$, the solution continuous beyond $T$. If $x_{1}\left(T_{-}\right)=\xi(T)$, we consider the problem:

$$
\begin{gathered}
x^{\prime}(t)=f(t, x(h(t))), \quad t>T \\
x(\sigma)=x_{1}(\sigma) \quad \sigma<T
\end{gathered}
$$




$$
\begin{gathered}
x(T)=\xi(T)+I(T, \xi(T)) \quad(\neq \xi(T)) \\
\left.\Delta x\right|_{x=\xi(t), t>T}=I(t, \xi(t)) .
\end{gathered}
$$

Under the hypothesis, this problem has a solution existing to the right of $T$. This procedure can be continued.

Theorem 5.2: Assume the hypotheses of Theorem 5.1 for $f$ and $h$. Suppose there are a finite number of $\xi_{i}$ 's at which impulses are given to the solutions. Let $\xi_{i}$ be continuous, $i=1,2, \ldots, m$. For any $i, j, 1 \leq i, j \leq m$, suppose that $i \neq j$ implies that

$$
\xi_{j}(t) \neq \xi_{i}(t)+I\left(t, \xi_{i}(t)\right) \text { for any } t \in \mathbb{R}_{+} \text {. }
$$

If $x_{0} \neq \xi_{i}(0)$ for any $i$, then the I.D.D.E. (4.1) to (4.3) has a unique solution $x \in P A C\left(\mathbb{R}_{+}\right)$.

Proof: Let $t \in[0, r], y\left(t, 0, x_{0}\right)$ be an absolutely continuous solution of the corresponding delay equation. Since $x_{0} \neq \xi_{i}(0)$, and as $\xi_{i}$ is continuous, there exists an interval $[0, \delta]$ such that $y\left(t, 0, x_{0}\right) \neq \xi_{i}(t)$ for all $t \in[0, \delta]$ for all $i=1,2, \ldots, m$. If $Y(t) \neq \xi_{j}(t)$ for any $j$ for all $t \in[0, r]$, take $x(t)=y\left(t, 0, x_{0}\right)$ for $t \in[0, r]$. If there exists $j$ such that for some $t_{1} \in(0, r], y\left(t_{1}\right)=\xi_{j_{1}}\left(t_{1}\right)$ and $y(t) \neq \xi_{i}(t)$ for any $t \in\left[0, t_{1}\right), i=1,2, \ldots, m$, we consider $y_{1}(t)=y\left(t, t_{1}, \xi_{j}\left(t_{1}\right)+\right.$ $\left.I\left(t_{1}, \xi_{j}\left(t_{1}\right)\right)\right), t \in\left[t_{1}, \infty\right)$. If $y_{1}(t) \neq \xi_{i}(t)$ for any $t \in\left[t_{1}, r\right)$ for any $i=1,2, \ldots, m$, then we take

$$
x(t)=\left\{\begin{array}{cc}
y(t), & t \in\left[0, t_{1}\right) \\
y_{1}(t), & t \in\left[t_{1}, r\right] .
\end{array}\right.
$$

If not, proceed as earlier to obtain $t_{2}, t_{3}, \ldots$ This process must terminate after a finite number of steps. Otherwise, we obtain a sequence $\left\{t_{n}\right\} \subseteq(0, r]$ such that

$$
y_{i}\left(t_{i}-\right)=\xi_{j_{i}}\left(t_{i}\right)
$$

and

$$
y_{i}\left(t_{i}\right)=\xi_{j_{i}}\left(t_{i}\right)+I\left(t_{i}, \xi_{j_{i}}\left(t_{i}\right)\right)
$$

for each $i$ and $y_{i}$ is absolutely continuous on $\left[t_{i}, t_{i+1}\right)$. We can assume, losing no generality, that $t_{i} \rightarrow t^{*} \leq r$ as $i \rightarrow \infty$. From the absolute continuity, we have 


$$
y_{i}\left(t_{i+1}\right)=y_{i}\left(t_{i}\right)+\int_{t_{i}}^{t_{i+1}} f(s, \varphi(h(s))) d s .
$$

From (5.7) and the continuity of $I$ and $\xi$, for any $c>0$, there exists a $\delta>0$ such that

$$
\left|\xi_{i}(s)-\xi_{j}(t)-I\left(t, \xi_{j}(t)\right)\right|>c>0
$$

for all $s, t \in\left(t^{*}-\delta, t^{*}\right]$. Hence choosing $i$ large enough for $t_{i}, t_{i+1} \in\left(t^{*}-\delta, t^{*}\right)$

$$
\begin{gathered}
0<c \leq\left|\xi_{n_{j}}\left(t_{i}\right)+I\left(t_{i}, \xi_{n_{i}}\left(t_{i}\right)\right)-\xi_{N-i+1}\left(t_{i+1}\right)\right| \\
=\left|y_{j_{i}}\left(t_{i}\right)-y_{n_{i}}\left(t_{i^{+}+1}^{+}\right)\right|=\left|\int_{t_{i}}^{t_{i+1}} f(s, \varphi(h(s))) d s\right| \\
\leq\left(\int_{t_{i}}^{t_{i+1}} p(s) d s\right) \cdot S(\|\varphi\|) .
\end{gathered}
$$

As $t_{i+1}-t_{i}$ can be made arbitrarily small, this leads to a contradiction.

Since the process terminates after a finite number of steps, the function $x$ defined as in (5.8) is a piecewise absolutely continuous solution of the I.D.D.E. on $[0, r]$. It is now easy from the estimates for $x$ to observe that the solution $x(t)$ can be extended to any interval $[0, k r], k$ being any positive integer.

\section{REFERENCES}

[1] Azbelev, N.V., Maksimov, V.P. and Rakhmatulina, L.F. (Russian), Introduction to the Theory of Functional Differential Equations, Nauka, Moscow, Russia 1991.

[2] Krishna, S.V. and Anokhin, A.V., Impulsive controllability of differential equations with deviating argument, Dynamical Systems and Applications, (to appear).

[3] Krishna, S.V., Anokhin, A.V., Impulsive controllability of equations with both retarded and advanced argument, (in preparation).

[4] Krishna, S.V., Control by impulse, delay and history, Proceedings of Dynamical Systems and Applications I, Dynamic Publishers, Atlanta, USA 1993 (to appear).

[5] Lakshmikantham, V. Bainov, D.D. and Simeonov, P.S., Theory of Impulsive Differential Equations, World Scientific Publishing Co., Singapore 1989.

[6] Lakshmikantham, V. and Leela, S., Differential and Integral Inequalities. Vol. I, Academic Press, New York 1969. 


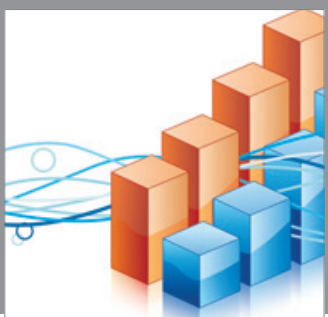

Advances in

Operations Research

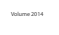

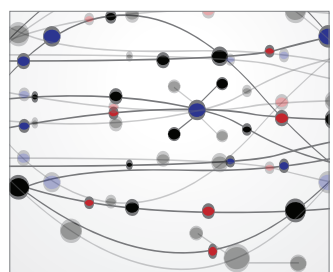

\section{The Scientific} World Journal
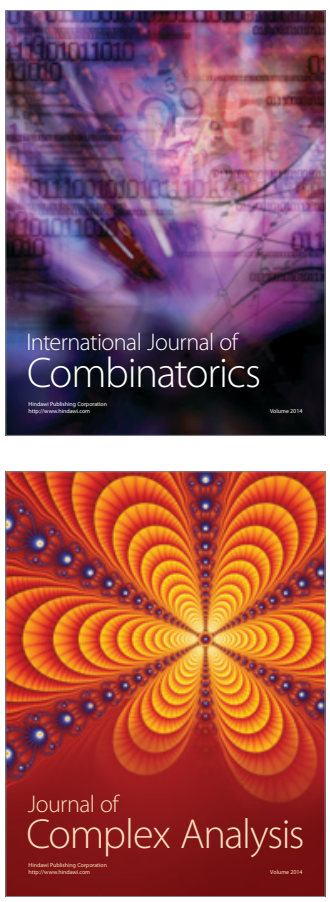

International Journal of

Mathematics and

Mathematical

Sciences
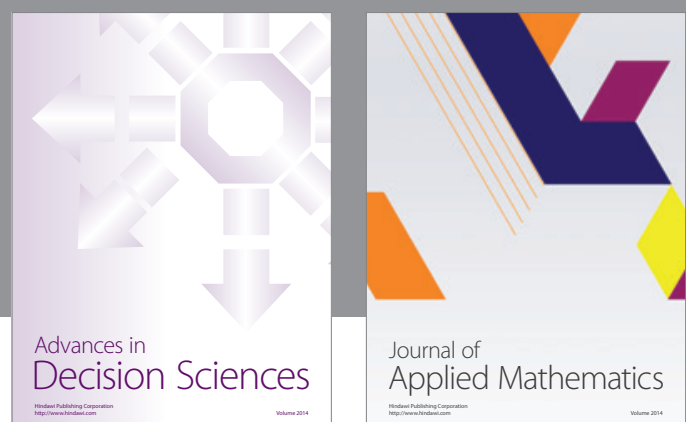

Journal of

Applied Mathematics
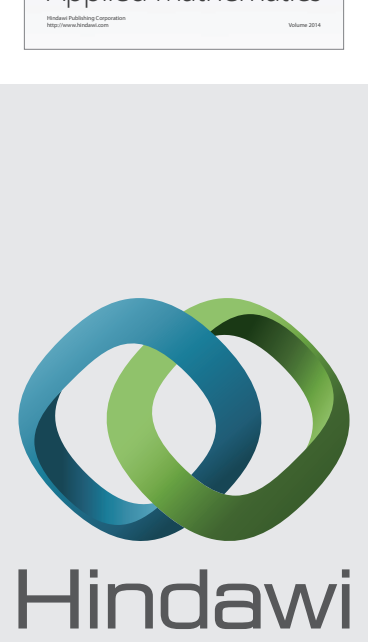

Submit your manuscripts at http://www.hindawi.com
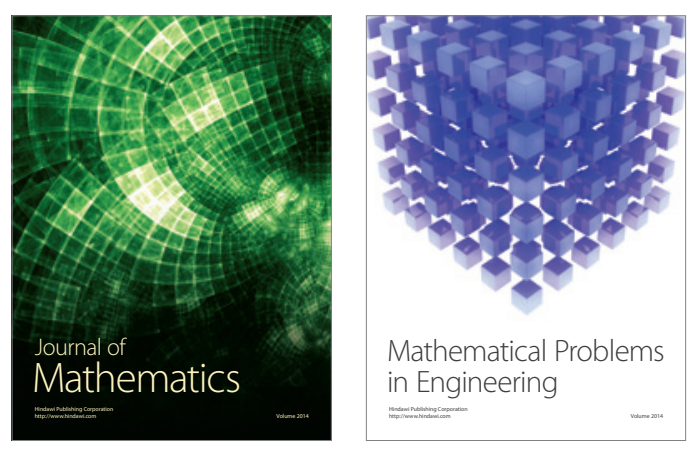

Mathematical Problems in Engineering
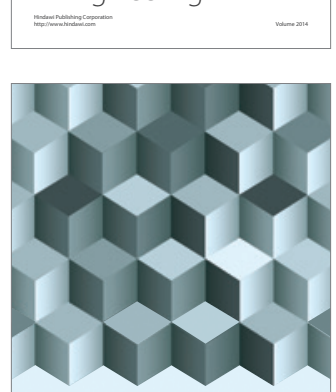

Journal of

Function Spaces
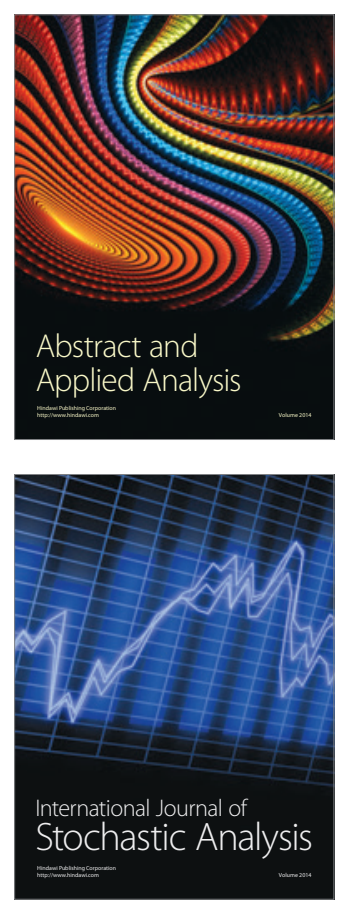

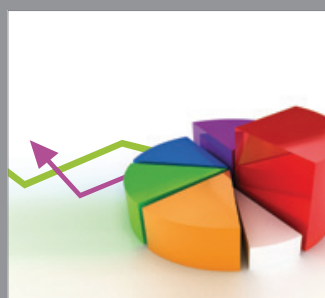

ournal of

Probability and Statistics

Promensencen
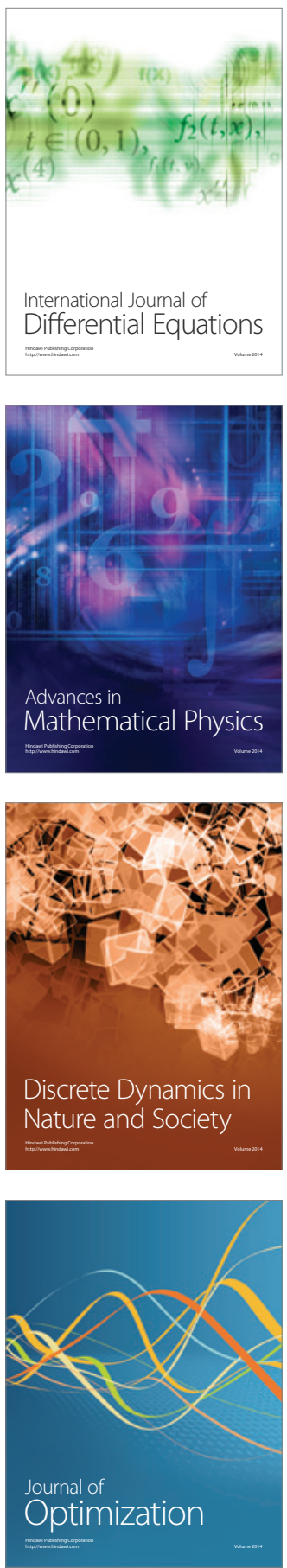